The tensile strength curves, Fig. 20, show values for this property which are nearly the same for all the compounds, probably within the experimental error due to slight differences in the state of cure. The same is true of the tensile values after accelerated aging. After the 60-day weathering test, however, it is observed that the stocks containing the larger proportions of zine oxide show definitely better aging qualities than those containing larger proportions of magnesium carbonate, as a result of the greater surface cracking tendency of rubber heavily compounded with magnesium carbonate. This again seems to be related in a degree to the crystalline character of the magnesium carbonate particles, since the plane surfaces and sharp edges of the crystals would be expected to favor the development of cleavage planes in the rubber itself when stressed, or perhaps even under the influence of changes in temperature, producing alternate expansion and contraction of the rubber matrix around each particle.

The hardness and permanent set (Fig. 21) of these compounds both show increasing values with increasing proportions of magnesium carbonate. The permanent set, however, does not exceed 16 per cent until half the zinc oxide has been replaced by magnesium carbonate.

The total resilient energy capacity and energy absorbed at 300 per cent elongation (Fig. 22 based on data in Table VI) both show a marked increase with successive increments of magnesium carbonate replacing zinc oxide in the compound. For the absorbed energy at 300 per cent elongation this increase is linear, as is also true of the curve showing stress at 300 per cent elongation for the same compounds (Fig. 20).

\title{
The Application of Ion Concentration Measurements to the Control of Industrial Processes
}

By Earl A. Keeler ${ }^{2}$

Leedos \& Northrup Co., Philadiliphia, Pennsylyania

$\mathrm{T}$

$\mathrm{HE}$ significance of ion concentrations has been the subject of many investigations during the last few years. The results obtained have demonstrated without question the unlimited possibilities existing for laboratory and industrial application of ion concentration measurements. It has been shown that in many instances biological activity, fermentation, and filtration are influenced to a large extent by variations in the $\mathrm{H}$-ion concentration, or reaction of the solutions involved. In addition, the way is open for improved control and accuracy in the many neutralization problems encountered in industrial chemical processes. Automatic control by means of motor driven valves and regulating apparatus has become a reality, and it is safe to predict that many troublesome problems of the present time will soon be handled automatically with great saving of. labor and expense.

The most widely known and most important of ion concentration measurements is that of the hydrogen ion. Many reactions occurring in colored or opaque solutions cannot be adequately governed by the use of color indicator. Quite frequently the hydrogen electrode is used to secure results that are impossible with the use of indicators. Another important asset lies in the ability of the hydrogen electrode to distinguish between actual acidity and total acidity. The controlling factor in reactions is usually the actual acidity, which depends directly upon the $\mathrm{H}$-ion concentration. Total acidity, as determined by titration, is a measurement of quantity or available acidity, and its effect upon reaction is indirect. An excellent example of the errors that one may be led into by depending upon total acidity measurement is found in the test of milk by titration with standard alkali. Milk of good quality, but containing a relatively large amount of acid salts, has often been rejected because of a high acidity as determined by titration. Another batch of milk of equal quality but containing less salts will exhibit a lower acidity when titrated. The actual acidity is largely due to the lactic acid content and can be measured by the $\mathrm{H}$-ion concentration.

In the past, the lack of suitable electrodes has greatly retarded the practical applications of $\mathrm{H}$-ion measurements

1 Received February 24, 1922.

Research Engineer, Leeds \& Northrup Co. to plant industrial problems. Fig. 1 shows a typical laboratory set-up consisting of a hydrogen electrode and a calomel electrode connected into a potentiometer circuit. One can readily realize the difficulties that would be encountered in applying such structures, or even radical modifications, to plant work. The calomel electrode at its best has often been a troublesome affair for unskilled hands to handle, and the hydrogen electrodes have lacked the requirement of convenient and rapid change of electrode surfaces. Contamination of hydrogen electrodes is usually unavoidable in most industrial processes. For this reason it is extremely important that the electrode elements may be easily removed and replaced.

Several new electrodes which are well suited for industrial work have been recently developed. These electrodes are shown in section in Fig. 2. It is to be noted that the structures are practically unbreakable, there being no glass parts used. The hydrogen electrode element is a disk of platinum gauze held in position by a knurled ring of hard rubber. A fresh electrode disk may be substituted for a used disk inside

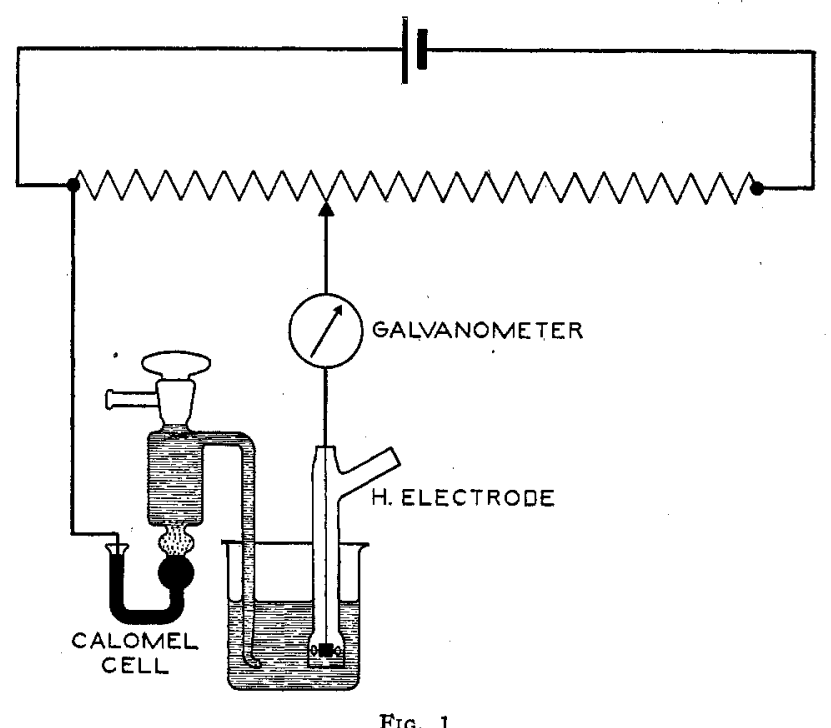

FIG. 1 


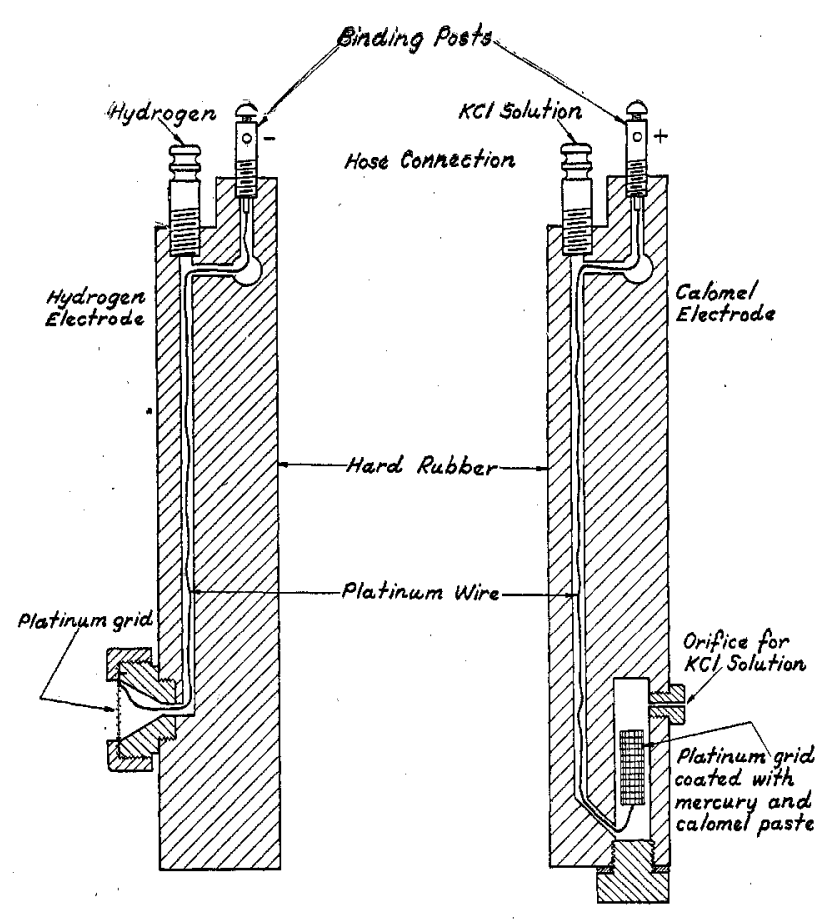

FiG. 2

of one minute. The calomel electrode is also unbreakable and contains no loose mercury. By coating a platinum grid with a paste of mercury and calomel, an electrode is secured that can be shipped ready for use. The only requirement is that a reservoir of potassium chloride solution be connected to supply the small flow of potassium chloride necessary to prevent diffusion of the solution under test into the calomel electrode. Fig. 3 shows the electrodes of Fig. 2 combined into one structure. The terminals, or binding posts, are shown at $\mathrm{E}$ and $\mathrm{F} . \mathrm{G}$ and $\mathrm{H}$ are the connections for hydrogen and

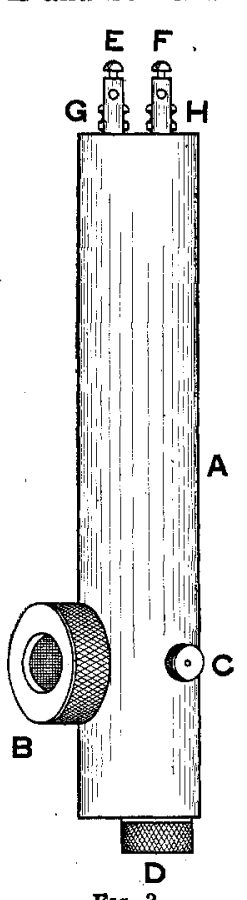

Fig. 3 the potassium chloride solution. The knurled ring holding the hydrogen electrode disk is shown at $B$. The orifice of the calomel electrode is the removable plug C. The knurled end plug $\mathrm{D}$ closes the calomel electrode chamber into which the paste-coated grid is mounted. The main body of the combined electrode, $A$, is made of hard rubber.

Another form which the electrode structure may take is shown in Fig. 4. Here a radical departure has been made from the usual method of applying the

A hydrogen gas to the electrode. Instead of bubbling the gas into the solution around the electrode, the flow of solution through a small aspirator is made to entrain the gas and direct it against the electrode disk. In this manner the gas is broken up into minute bubbles and distributed through the solution. Such an electrode has been found very satisfactory for continuous flow applications, such as the recording and control of boiler feed water acidity. A small glass wool filter connected as in Fig. 4 will greatly increase the period over which an electrode disk may be used before removal for cleaning and replatinizing is necessary. A change of the hydrogen electrode disks every two or three days is advisable, although experience has shown that such electrode will sometimes operate satisfactorily for several weeks without cleaning.

The hydrogen-ion method is particularly well adapted to the control of neutralization processes and the measurement of low acidity or alkalinity. Fig. 5 shows a reproduction of a record produced by an automatic hydrogen-ion recorder during the reaction of sodium hydroxide solution with hydrochloric acid solution. It will be noted that the greatest change of voltage for a given change in acidity takes place around the neutral point. The ordinate of this record is marked "time" and may be taken as proportional to change of acidity or alkalinity. The records shown in Figs. 6, 7, 8, 9, and 10 are typical of the results that are secured upon application of the hydrogen-ion recorder to many industrial problems.

The carbonation of beetsugar juice is shown in the record of Fig. 6. Milk of lime had been added to this juice prior to the carbonation. It will be noted that the curve is practically flat (1.05 volts) during the first portion of the carbonation. Then a break occurs, producing a "knee" in the curve. During the remainder of the carbonation the slope of the curve is very appreciable, thus giving considerable change in voltage for small changes in alkalinity. Three samples were taken between the "knee" of the curve and the neutral point. The titration of these samples was made with $0.036 \mathrm{~N}$ sulfuric acid and the results expressed in grains of calcium oxide per $100 \mathrm{cc}$. of solution. Phenolphthalein was used as an indicator.

Fig. 7 shows the record secured during the neutralization of pineapple juice. Milk of lime is added to the juice until the neutral point is reached. Phenolphthalein tests showed that 0.75 volt corresponded to the real neutral point.

An interesting application of the recorder is shown in Fig. 8. The changes of alkalinity occurring when tooth paste goes into water solution are of importance because of the necessity for proper neutralization of the reaction known as "acid

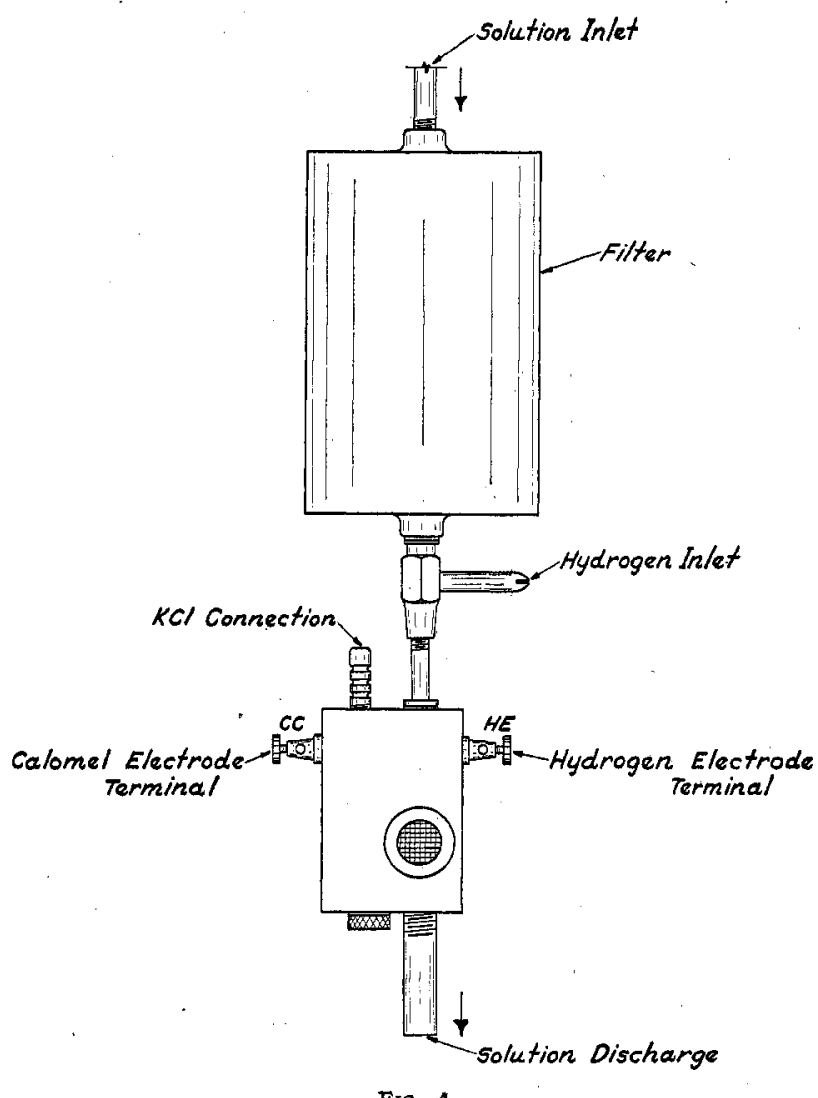

FIG. 4 
mouth." The shape of the curve shows that the alkalinity becomes practically constant after the first 2 or 3 minutes.

A peculiar record is reproduced in Fig. 9. The alkalinity

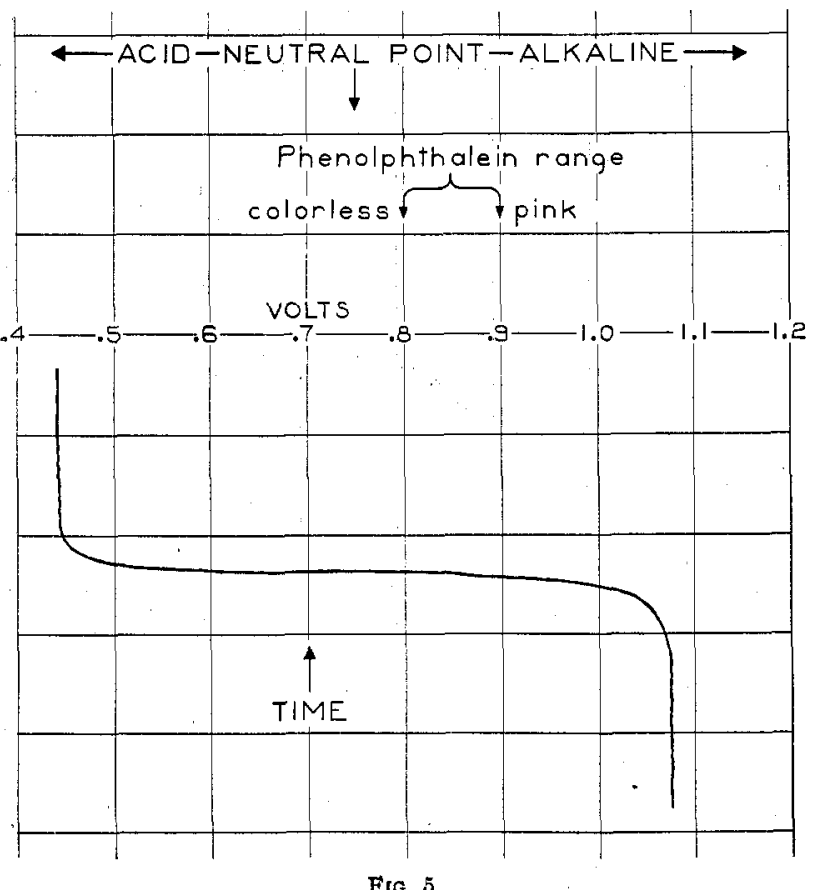

of wool scouring liquor is difficult to determine because of the color and suspended matter of all kinds. A titration of some of this liquor with sulfuric acid resulted in the record of Fig. 9. The liquor contains some free alkali in the form of soda ash, some soap made up of red oil, soda ash, and the solution constituents of the greasy wool containing complex organic acids combined with potash. It seems that there are three steps involved in the neutralization: (1) the neutralization of the free alkali; (2) the decomposition of the red oil soaps; and (3) the liberation of a gas caused by the decomposition of the potash-organic acid compounds.

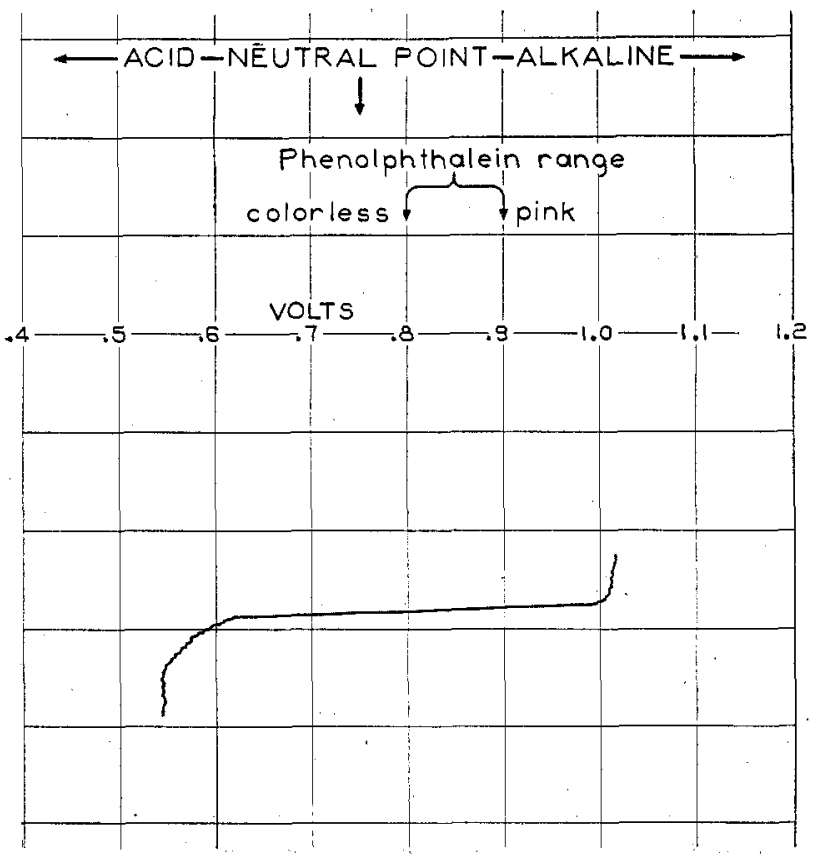

FiG. 7
Another interesting feature is shown in Fig. 10, and here the two end-points of the reaction of phosphoric acid with sodium hydroxide indicate the formation of the primary and secondary phosphates.

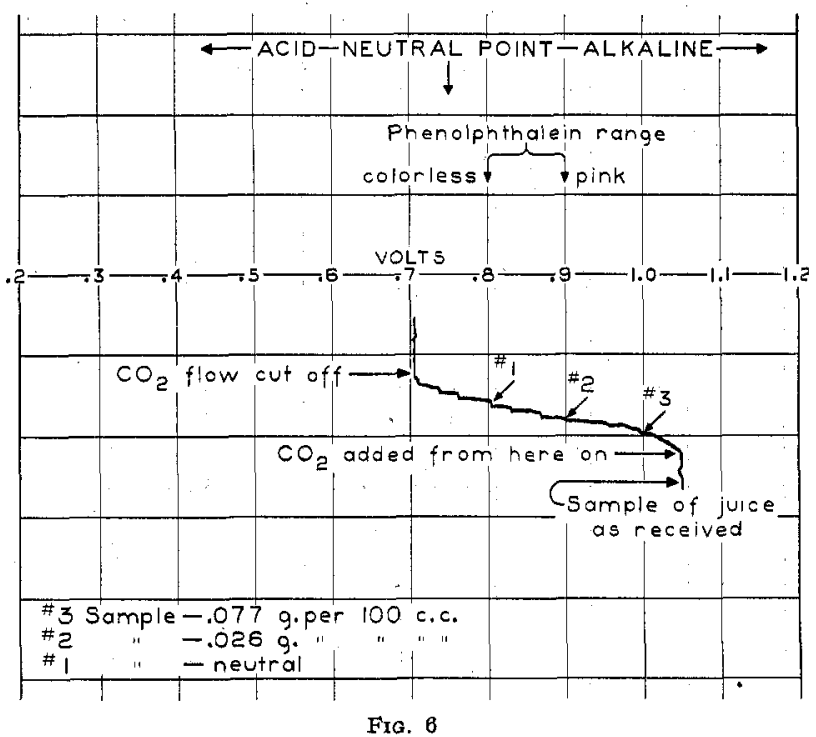

These are only representative of the many possibilities existing for the application of ion concentration methods. Several comprehensive papers have recently been published on the subject of $\mathrm{H}$-ion measurements as applied to filtration problems in water purification ${ }^{3}$ and sewage disposal. ${ }^{4}$ Another subject upon which a great deal of interesting work has been done, is that concerning the effect of the hydrogenion concentration upon the properties of tanning liquors used in the leather industry. ${ }^{5}$

The step from recording apparatus, such as that shown in Fig. 11, to equipment for automatic control is a natural one to undertake. Frequently, the advantages to be gained by automatic control are far greater than those secured by re-

- Abel Wolmand and Frank Harman, Chem. Met. Eng, 25 (1921), 502.

- J. A. Wilson and H. M. Heisig, Tims Journal, 13 (1921), 406.

J. A. Wilson and Erwin J. Kern, Ibid., 13 (1921), 1025.

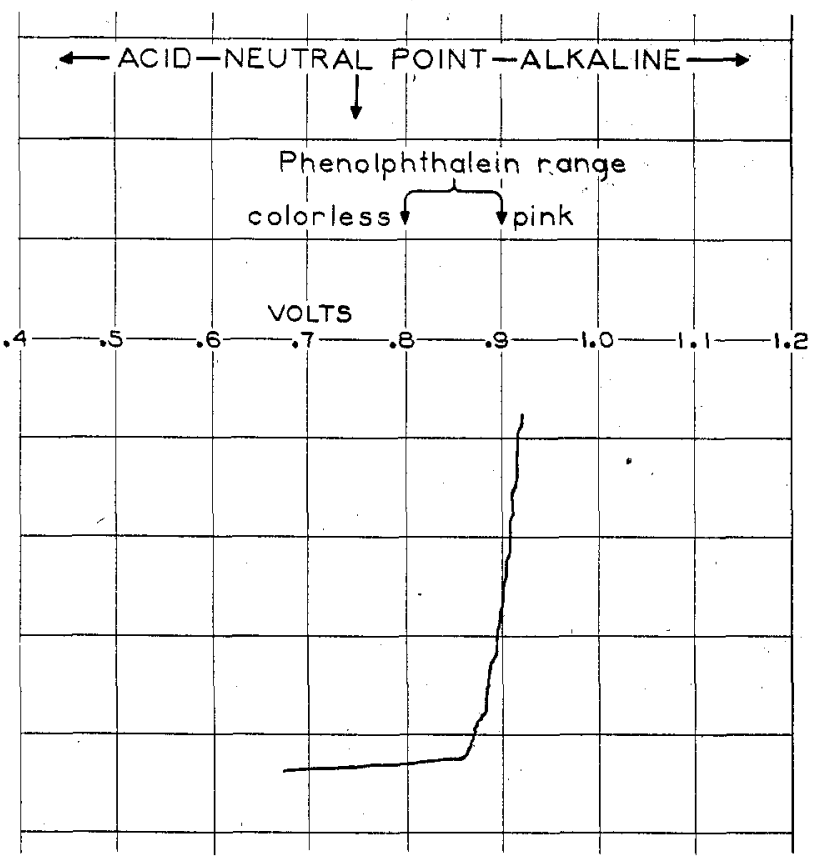

Fig. 8 